\title{
Tumor-like Presentation of Primary Angiitis of the Central Nervous System. Report of Two Cases
}

\author{
Santamaría José Ignacio ${ }^{1 *}$, Mormandi Rubén ${ }^{1}$, Cervio Andrés ${ }^{1}$, Condomi Alcorta Santiago ${ }^{1}$, Virginia Andrea \\ Pujol Lereis*2 and Ameriso Sebastián Francisco ${ }^{2}$ \\ ${ }^{1}$ Department of Neurosurgery, Institute for Neurological Research - FLENI, Argentina \\ ${ }^{2}$ Department of Neurology, Institute for Neurological Research - FLENI, Argentina \\ Submission: Mrach 17, 2017; Published: April 27, 2017 \\ *Corresponding author: Pujol Lereis Virginia Andrea, Department of Neurology, Institute for Neurological Research, FLENI, Montañeses 2325 \\ (C1428AQK), Buenos Aires, Argentina, Tel: +5411-5777-3200; Fax: +5411-5777-3200; Email: vpujol@fleni.org.ar
}

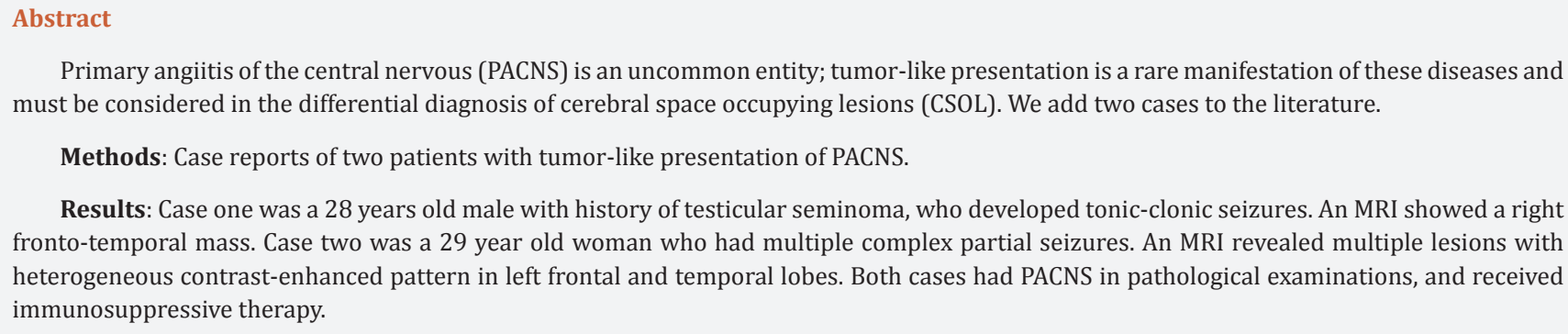

Primary angiitis of the central nervous (PACNS) is an uncommon entity; tumor-like presentation is a rare manifestation of these diseases and must be considered in the differential diagnosis of cerebral space occupying lesions (CSOL). We add two cases to the literature.

Methods: Case reports of two patients with tumor-like presentation of PACNS.

Results: Case one was a 28 years old male with history of testicular seminoma, who developed tonic-clonic seizures. An MRI showed a right fronto-temporal mass. Case two was a 29 year old woman who had multiple complex partial seizures. An MRI revealed multiple lesions with heterogeneous contrast-enhanced pattern in left frontal and temporal lobes. Both cases had PACNS in pathological examinations, and received immunosuppressive therapy.

Conclusion: PACNS should be considered in the differential diagnosis of CSOL. Awareness of this presentation may facilitate early management.

Keywords: Primary central nervous system vasculitis; Central nervous system; Angiitis; Immunosuppressive therapy; Vascular inflammatory diseases

\section{Introduction}

Primary angiitis of the central nervous system (PACNS) is a rare condition of unknown etiology affecting the central nervous system (CNS) in the absence of systemic vasculitis, inflammatory or infectious disease [1,2]. Initially described in 1959 by Cravioto \& Feigin [3], this condition has been increasingly reported in the literature. It is characterized by nonspecific granulomatous or nongranulomatous inflammation of small and/or medium caliber cerebral, leptomeningeal, and/or spinal cord arteries and veins [3]. Clinical presentations are often nonspecific, with diagnosis based on clinical, laboratory, imaging and pathological findings4. It generally occurs in young-adults (30-60 years old) without vascular risk factors, with a mean age of presentation of 45.5 years. The most common manifestations are headaches, focal neurologic symptoms, seizures, cognitive impairment, and psychiatric changes [1]. It has an estimated incidence of 1-2.4 per million per year in European populations [2-5]. The tumormimicking form is rare, accountry for 5 to $15 \%$ of all cases of PACNS [5-7]. Tumor-mimicking PACNS often carries worse outcomes than the typical forms [8].
Diagnosis of PACNS represents a difficult challenge for clinicians due to the lack of clinical, serological, and neuroradiological specific hallmarks. Diagnostic criteria for PACNS were proposed in 1988 by Calabrese \& Mallek [1]. These criteria have been used widely for clinical and investigation purpose. Patients should meet all of the following criteria to be diagnosed with PACNS:

A. An acquired otherwise unexplained neurological or psychiatric deficit,

B. Either classic angiographic or histopathological features of angiitis within the CNS, and

C. No evidence of systemic vasculitis or any disorder that could cause or mimic the angiographic or pathological features of the disease.

Prompt and accurate diagnosis of PACNS is essential to implement specific treatments. An under-recognized presentation of PACNS can be seen in patients that have mass 
lesions mimicking space occupying processes. The aim of this work was to report two cases of PACNS mimicking a brain tumor.

\section{Method}

Retrospective review of medical records and diagnostic tests of two patients with PACNS mimicking a brain tumor. The Results diagnosis of PACNS was obtained by histological confirmation. Variables collated included demographic variables, clinical features, results of relevant investigations, therapeutic interventions, and clinical outcomes. The ethics committee of the institution approved the study.

\section{Case 1}
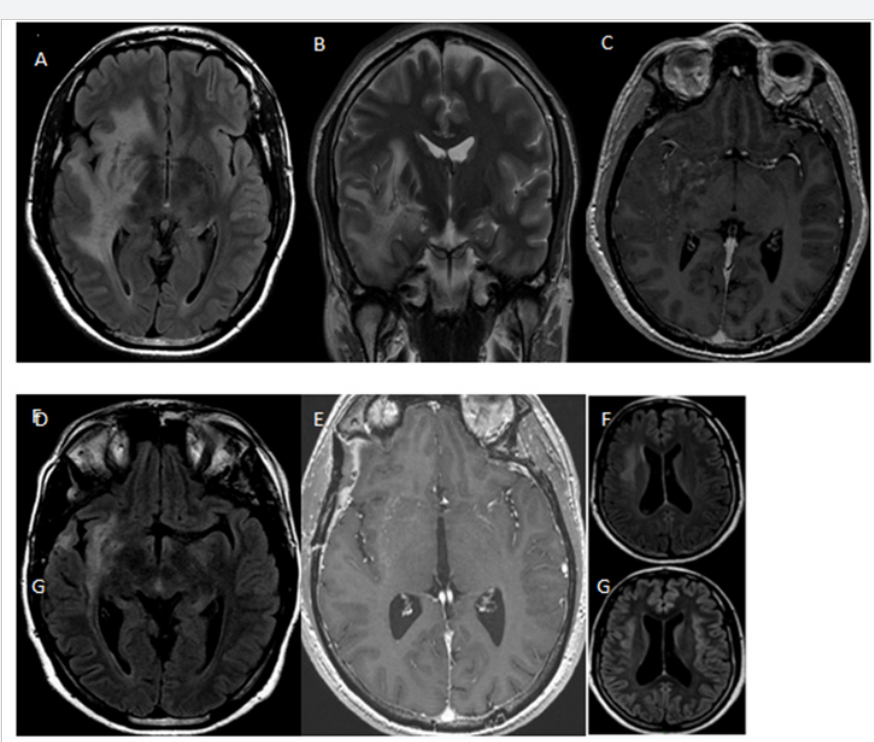

Figure 1: Initial and post operative MR image from case 1. Preoperative MR images showing right fronto-temporo-insular lesion with irregular contrast enhancement: (A) axial fluid-attenuated inversion recovery (FLAIR), (B) coronal T2-weighted, (C) T1 weighted. Six months postoperative MR (D) axial FLAIR and (E) T2 weighted images showing improvement of previous lesion. (F) Fourteen months postoperative MR axial FLAIR image showing new right frontal lesion. (G) Twenty months postoperative MR axial FLAIR image showing improvement of new lesion.

A 28 year old man with history of testicular seminoma surgically removed in April 2012. He had a tonic-clonic seizure in April 2013 with full recovery. Magnetic resonance imaging (MRI) showed a right fronto-temporal space occupying lesion (Figure 1). Tumor single and multi-voxel MR spectroscopy showed elevate choline (Cho) and glutamine, with decline in $\mathrm{N}$-acetyl-aspartate (NAA). Digital subtraction angiography was normal. Erythrocyte sedimentation rate (ESR) was $40 \mathrm{~mm} / \mathrm{h}, \mathrm{C}$-reactive protein was $19.9 \mathrm{mg} / \mathrm{dL}$. Blood blood count, metabolic profile, thyroid profile, and immunoelectrophoretic proteinogram were normal. Human immunodeficiency virus (HIV), hepatitis A, B and C panel, VDRL, rheumatologic studies (C3-C4, c/p-anti-neutrophil cytoplasmic antibody, anti-nuclear antibody, rheumatoid factor), and Chagas IgG and IgM were all negative. With a preliminary diagnosis of brain tumor, a biopsy was performed. Through a pterional approach, a hard-woody gray lesion with minimal bleeding was observed. The pathological study reported CNS vasculitis with perivascular lymphocytic inflammatory infiltrate, isolated mononuclear giant cells, isolated immunostaining but not representative CD3-20, isolated intraparietal granuloma and reactive gliosis. Polymerase chain reaction (PCR) tested in brain tissue for varicella-zoster virus (VZV), herpes simplex virus
1 and 2 (HSV-1 and HSV-2), JC virus (JCV), virus epstein-barr (VEB), toxoplasmosis and Whippel's disease were all negative. Congo red stain was negative.

The final diagnosis was PACNS. Patient received high-dose of intravenous methylprednisolone for five days and then was discharged with oral prednisone $1 \mathrm{mg} / \mathrm{kg} /$ day for six months and posterior tappering. During follow up he had a good initial clinical and radiological evolution (Figure 1). Fourteen months after biopsy an MRI revealed an hiperintense temporo-insular asymptomatic lesion interpreted as disease recurrence. High dose oral prednisolone and monthly intravenous cyclophosphamide were given. A repeat brain MRI after 6 months of treatment demostrated lesion remission (Figure 1).

\section{Case 2}

A 29 year old woman without any significant past medical history had several complex partial seizures. Her post ictal neurological examination was normal. MRI revealed multiple hyperintense lesions with contrast-enhanced T1-weighted heterogeneous pattern in left temporal and frontal lobes (Figure 2). Single and multi-voxel spectroscopy showed elevated Cho. Blood laboratory tests were normal including thyroid profile, 


\section{Open Access Journal of Neurology \& Neurosurgery}

HIV, HCV, HBV, VDRL, ANCA C, ANCA P, Toxoplasmosis IgG and IgM, and Brucellosis IgG and IgM. Cerebrospinal fluid (CSF) was normal, PCR for enterovirus, tuberculosis, and VDRL were all negative. With a presumption of brain tumor, a left fronto-basal lesion biopsy was performed through a supraorbital lateral approach. A grayish lesion with minimal bleeding was observed.

Pathological examination reported moderate perivascular lymphocytic infiltration and reactive gliosis; 3-20 CD markers were not representative. PCR tested in brain tissue for VZV, HSV- 1-2-6-8, JCV, VEB, citomegalovirus (CMV) were all negative.
Congo red stain was negative. These findings were consistent with a diagnosis of PACNS. The patient was first treated with high-dose of intravenous methylprednisolone for five days and then continued with oral prednisone $1 \mathrm{mg} / \mathrm{kg} /$ day for six months and posterior tappering. Fourteen months after diagnosis she had increased seizure frequency associated with a new left temporal lobe lesion. She received rituximab, $2 \mathrm{gr}$ total doses by infusions of $1 \mathrm{gr}$ separated by 14 day. After 9 months of rituximab treatment, she had good outcome, with seizure and lesion remission (Figure 2).
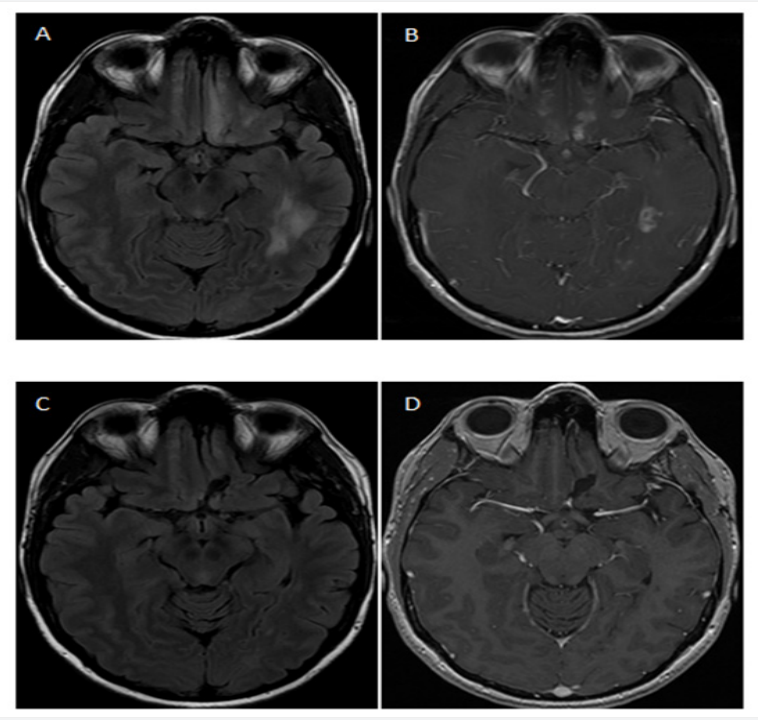

Figure 2: Initial and post operative MR image from case 2. Preoperative MR images showing left straight girus, subcortical left frontal and temporal lesions with irregular contrast enhancement: (A) axial fluid-attenuated inversion recovery (FLAIR), (B) T1-weighted. Nine months post rituximab treatment: (C) T2 weighted MR axial image showing absence of the previous lesions; (D) T1 weighted MR image without gadolinium enhancement in previuos pathological sites.

\section{Discussion}

We report 2 cases of pathology confirmed tumor-like PACNS. PACNS is a rare disease of variable presentations and unclear etiology. Clinical presentations of PACNS are protean and often non-specific, with differential diagnoses including malignancy, infection and other connective tissue disease [1,9]. Tumour-like mass lesions are rare, seen in only $5-15 \%$ of patients, and are generally clinically and radiologically indistinguishable from mass lesions of other etiologies [5-7].

Brain biopsy remains the gold standard to establish a diagnosis of PACNS, ideally with an open wedge biopsy of a discrete lesion including grey and white matter, leptomeninges and cortical vessels $[10,11]$. It typically shows vasculocentric transmural inflammation with granulomatous, lymphocytic or necrotizing infiltration of small - medium parenchymal or leptomeningeal vascular wall [12]. Nevertheless, segmental involvement results in false negative rates up of to $25 \%$ [13]. The diagnostic yield increases when radiologically affected areas are targeted and when including leptomeninges in the biopsy. Our patients illustrate that histological diagnosis is crucial as both subjects had a preliminary diagnosis of brain tumor.

Brain CT scans, MRI, magnetic resonance angiography (MRA), and conventional angiography are all useful diagnostic imaging modalities. MRI usually demonstrated acute findings considered with sensitivity of $90 \%$ or better, but findings such as parenchymal or leptomeningeal enhancement, cerebral infarction, intracranial haemorrhage and T2-weighted or fluidattenuated inversion recovery (FLAIR) hyperintensities are not pathognomoni $[8,14]$. The most common finding is infarction in $53-97 \%$ of patients, multifocal and/or bilateral in over $80 \%$ [2-15]. In the few reports of neuroimaging features of tumormimicking PACNS and in our cases, MRI did not show brain infarction, and such patients are often misdiagnosed as high grade gliomas because of gadolinium enhancement, T2-weighted hyperintensity, T1-weighted hypointensity, haemorrhage and significant oedema and mass effect $[6,16,17]$.

There are a few reports of MR spectroscopy features of PACNS $[16,18,19]$. Elevation of choline/NAA ratio and lipid-lactate peaks 
in varying degree, nonspecific finding, was previously observed, as noted in our cases. In one study, authors additionally observed marked elevation of glutamate peaks in agreement with our case 118. The role of spectroscopy in the diagnosis of pseudotumoral PACNS deserves further investigations. Digital subtraction angiography can reveal typical signs of vasculitis, but it is normal in up to $40 \%$ of patients, especially those with involvement of small vessels $[1,16]$. Nonspecific angiographic findings in PACNS include tapering of the vessel lumen of a single or many vessels and fusiform arterial dilatations, multifocal vascular occlusions, development of collateral circulation, or delayed contrastmedium enhancement and washout time [1]. Cerebral digital angiography has low sensitivity and specificity for the diagnosis of PACNS and should be interpreted with caution.

Cerebral amyloid angiopathy (CAA) may cause vascular inflammatory reaction which can be indistinguishable from PACNS. There are reports of tumor-mimicking presentation in patients with SNC angeitis associated with B-amyloid deposits [1]. This emphasizes the need to stain for amyloid protein in patients presenting with tumor-like PACNS. Furthermore, CAA pseudotumoral lesions are less respondent to immunosuppressive therapy [1].

CSF study is a vital diagnostic tool to identify infection, malignancy, and multiple sclerosis, and should be performed on every patient suspected to have PACNS unless it is contraindicated. The CSF findings in $80-90 \%$ of biopsy-proven PACNS cases include a lymphocyte-predominant pleocytosis, elevated protein, and normal glucose level, an inflammatory pattern consistent with aseptic meningitis $[4,5,14]$. Cultures and serologies for infections should be performed in all patients. In case 2, CSF was normal. There are no controlled trials evaluating the best treatment strategy for pseudotumoral PACNS. In patients with PACNS presenting as mass lesions, prompt recognition and aggressive immunosuppressive therapy appears to be associated with favourable outcomes and may obviate surgical excision6. Good response rates to therapy are seen with early diagnosis and immunosuppressive therapy, particularly high dose corticosteroids combined with intravenous cyclophosphamide $[16,17,20,21]$. Limited data are available for the use of rituximab in PACNS [22,23]. This drug must be reserved for patients intolerant to conventional immunodepressive agents or as secondline for refractory cases.

In summary, spare occupying effect is a rare presentation of PACNS. Neuroimaging features of tumor mimicking PACNS do not allow differentiation from other conditions including malignancy at initial presentation. Biopsy is the confirmatory test of choice and should be performed prior initiation of therapy. Aggressive immunosuppressive treatment is associated with favorable outcomes. PACNS should be considered in the differential diagnosis of brain tumors. Awareness of this presentation may facilitate more prompt diagnosis and treatment.

\section{Conflict of Interest}

The authors certify that they have No affiliations with or involvement in any organization or entity with any financial interest (such as honoraria; educational grants; participation in speakers' bureaus; membership, employment, consultancies, stock ownership, or other equity interest; and expert testimony or patent-licensing arrangements), or non-financial interest (such as personal or professional relationships, affiliations, knowledge or beliefs) in the subject matter or materials discussed in this manuscript.

\section{References}

1. Hajj-Ali RA, Singhal AB, Benseler S, Molloy E, Calabrese LH (2011) Primary angiitis of the CNS. Lancet Neurol 10: 561-572.

2. Scolding NJ, Wilson H, Hohlfeld R, Polman C, Leite I, et al. (2002) EFNS Cerebral Vasculitis Task Force. The recognition, diagnosis and management of cerebral vasculitis: a European survey. Eur J Neurol 9(9): 343-347.

3. Cravioto H, Feigin I (1959) Noninfectious granulomatous angiitis with a predilection for the nervous system. Neurology 9: 599-609.

4. Calabrese LH, Furlan AJ, Gragg LA, Ropos TJ (1992) Primary angiitis of the central nervous system: diagnostic criteria and clinical approach. Cleve Clin J Med 59: 293-306.

5. Salvarani C, Brown RD, Calamia KT, Christianson TJ, Weigand SD, et al. (2007) Primary Central Nervous System Vasculitis: analysis of $101 \mathrm{~Pa}-$ tients. Ann Neurol 62: 442-451.

6. Molloy ES, Singhal AB, Calabrese LH (2008) Tumour-like mass lesion: an under-recognised presentation of primary angiitis of the central nervous System. Ann Rheum Dis 67(12): 1732-1735.

7. Abdel Razek AA, Alvarez H, Bagg S, Castillo M (2014) Imaging spectrum of CNS vasculitis. Radiographics 34(4): 873-894.

8. Birnbaum J, Hellman DB (2009) Primary angiitis of the central nervous system. Arch Neurol 66(6): 704-709.

9. Hajj-Ali RA, Calabrese LH (2014) Diagnosis and classification of central nervous system vasculitis. J Autoimmunity 48-49: 149-152.

10. Parisi JE, Moore PM (1994) The role of biopsy in vasculitis of the central nervous system. Semin Neurol 14: 341-348.

11. Alrawi A, Trobe JD, Blaivas M, Musch DC (1999) Brain biopsy in primary angiitis of the central nervous system. Neurology 53(4): 858-860.

12. Giannini C, Salvarani C, Hunder G, Brown RD (2012) Primary central nervous system vasculitis: pathology and mechanisms. Acta Neuropathol 123(6): 759-772

13. Cupps TR, Moore PM, Fauci AS (1983) Isolated angiitis of the central nervous system. Prospective diagnostic and therapeutic experience. Am J Med 74(1): 97-105

14. Younger DS (2004) Vasculitis of the nervous system. Curr Opin Neurol 17: 317-336.

15. Geri G, Saadoun D, Guillevin R, Crozier S, Lubetzki C, et al. (2014) Central nervous system angiitis: a series of 31 patients. Clin Rheumatol 33(1): 105-110.

16. Lee Y, Kim JH, Kim E, Park SH, Yim YJ, et al. (2009) Tumor-mimicking primary angitis of the central nervous system: initial and follow-up MR features. Neuroradiology 51(10): 651-659

17. You G, Yan W, Zhang W, Li S, Li G, et al. (2011) Isolated angiitis of the central nervous system with tumor-like lesion, mimicking brain ma- 
lignant glioma: a case report and review of the literature. World J Surg Oncol 9: 97-100.

18. Panchal NJ, Niku S, Imbesi SG (2005) Lymphocytic vasculitis mimicking aggressive multifocal cerebral neoplasm: mr imaging and MR spectroscopic appearance. Am J Neuroradiol 26: 642-645.

19. Beppu T, Inoue T, Nishimoto H, Nakamura S, Nakazato Y, et al. (2007) Primary granulomatous angiitis of the central nervous system: findings of magnetic resonance spectroscopy and fractional anisotropy in diffusion tensor imaging prior to surgery. Case report. J Neurosurg 107(4): 873-877.

20. Fauci AS, Haynes B, Katz P (1978) The spectrum of vasculitis: clinical, pathologic, immunologic and therapeutic considerations. Ann Intern
Med 89(part 1): 660-676.

21. Oon S, Roberts C, Gorelik A, Wicks I, Brand C (2013) Primary angiitis of the central nervous system: experience of a Victorian tertiary-referral hospital. Intern Med J 43(6): 685-692.

22. De Boysson H, Arquizan C, Guillevin L, Pagnoux C (2013) Rituximab for primary angiitis of the central nervous system: report of 2 patients from the French COVAC cohort and review of the literature. J Rheumatol 40(12): 2102-2103.

23. Salvarani C, Brown RD, Huston J, Morris JM, Hunder GG (2014) Treatment of primary CNS vasculitis with rituximab: case report. Neurology 82: $1287-1288$.

\section{Your next submission with Juniper Publishers will reach you the below assets}

- Quality Editorial service

- Swift Peer Review

- Reprints availability

- E-prints Service

- Manuscript Podcast for convenient understanding

- Global attainment for your research

- Manuscript accessibility in different formats

( Pdf, E-pub, Full Text, Audio)

- Unceasing customer service

Track the below URL for one-step submission https://juniperpublishers.com/online-submission.php 